\title{
Cost-effectiveness of the NICE guidelines for screening for latent tuberculosis infection: the QuantiFERON-TB Gold IGRA alone is more cost- effective for immigrants from high burden countries
}

\author{
A B Hardy, R Varma, T Collyns, S J Moffitt, C Mullarkey, J P Watson
}

Leeds Teaching Hospitals NHS Trust, Leeds, UK

\section{Correspondence to} Dr A B Hardy, Leeds Teaching Hospitals NHS Trust, Department of Respiratory Machine, Beckett Street, Leeds LS9 7TF, UK;

andrewbhardy@yahoo.co.uk

Received 14 May 2009

Accepted 11 November 2009

Published Online First

8 December 2009

\section{ABSTRACT}

NICE (National Institute for Health and Clinical Excellence) guidelines for new entrant tuberculosis (TB) screening recommend chest $x$ ray (CXR) for immigrants from countries with TB incidence $>40 / 10^{5}$, and tuberculin skin test (TST) for people with normal CXR from very high TB prevalence countries. A revised screening policy using first-line QuantiFERON-TB Gold (QFT) in high risk immigrants was piloted in 2007. Initially, TST was offered to immigrants from countries with TB incidence $200-339 / 10^{5}$, and OFT to those from countries with incidence $>340 / 10^{5}$. When increased resources became available, all immigrants from countries with TB incidence $>200 / 10^{5}$ had QFT. Those with positive QFT were invited for CXR. 1336 immigrant were invited for screening, with a $32 \%$ attendance rate. 280 patients had QFT, of which $38 \%$ were positive, with $<2 \%$ being indeterminate. Using the NICE approach, the cost of screening these 280 immigrants would be $£ 13346.75$ (£47.67 per immigrant) and would identify 83 cases of latent TB infection (LTBI). Using first-line QFT followed by CXR the cost was $£ 9781.82$ (£34.94 per immigrant) and identified 105 cases of LTBI. The cost to identify one case of LTBI following NICE guidelines would be $£ 160.81$ and using the present protocol was $£ 93.16$. For immigrants from high risk countries QFT blood testing followed by CXR is feasible for TB screening, cheaper than screening using the NICE guideline and identifies more cases of LTBI.

\section{INTRODUCTION}

Notifications of new cases of tuberculosis (TB) have increased over the last 10 years in the UK. This is due to an increase in TB in foreign-born individuals. ${ }^{1}$ Effective control of TB requires identification of active and latent TB in populations arriving from high risk countries. Traditionally, screening has been based on chest $x$ ray (CXR) and tuberculin skin testing (TST). QuantiFERON-TB Gold (OFT) is a newer test for TB, based on the quantification of interferon $\gamma$ released from lymphocytes in whole blood sensitised to specific TB antigens ESAT-6 and CFP-10. Advantages over TST include higher specificity and sensitivity and the need for only one contact with a healthcare professional

There is a moderate agreement between TST and OFT. $^{2} 3$ A study of Dutch army recruits who had been deployed in high risk countries showed an $82 \%$ concordance between TFT and OFT, with a high discordance between TST-positive OFT-negative individuals. ${ }^{4}$ This suggests that latent TB infection may be overdiagnosed if there is an over-reliance on TST. This is because TST can be positive in people with prior BCG vaccination and environmental mycobacteria. OFT has been shown to reduce screening costs in TB contacts. ${ }^{5}$ Another commercially available interferon $\gamma$ assay, T-SPOT.TB, which shows good agreement with OFT, ${ }^{6}$ has been shown to be cost-effective in screening $\mathrm{TB}$ contacts. ${ }^{7}$ Studies have suggested that a TST followed by a T-SPOT.TB protocol is cost-effective for contact screening for latent TB infection. ${ }^{8}$ There are no published data regarding the use of either interferon $\gamma$ release assay (IGRA) for immigrant screening

In the UK, National Institute for Health and Clinical Excellence (NICE) guidelines for new entrant TB screening currently recommend CXR for immigrants from countries with $\mathrm{TB}$ incidence $>40 / 10$, and TST for people with normal CXR from very high TB incidence countries. ${ }^{9}$ An IRGA is currently recommended for those with positive TST and normal CXR to confirm the diagnosis of latent TB infection (LTBI). The purpose of this study was to assess the cost-effectiveness of first-line OFT for screening immigrants from high risk countries, a strategy which has not been appraised in the current NICE guidelines due to a lack of information regarding cost-effectiveness in the patient group.

\section{METHODS}

The Leeds TB Screening Service serves a population of 750000 . Due to cost and personnel issues, we are unable to screen all new immigrants from countries with TB incidence $>40 / 10^{5}$. Previous audit in the department has demonstrated that the majority of cases of TB in this region are found if a threshold of $200 / 10^{5}$ is used. Therefore, since 2005 our policy has been to offer screening to immigrants from countries with TB incidence $>200 / 10^{5}$. A revised screening policy was introduced on 1 January 2007. Initially, TST was offered to immigrants from countries with TB incidence $200-339 / 10^{5}$, and OFT to those from countries with incidence $>340 / 10^{5}$. When increased resources became available, all immigrants from countries with $\mathrm{TB}$ incidence $>200 / 10^{5}$ had OFT. Those with positive OFT were invited for CXR.

TFT was carried out using 2 tuberculin units (TU) of purified protein derivative on the volar aspect of the forearm, according to the Mantoux 
method. Cut-off for a positive result was $6 \mathrm{~mm}$ in those without prior BCG and $15 \mathrm{~mm}$ in those with prior BCG. OFT was performed according to the manufacturer's instructions using 1 $\mathrm{ml}$ aliquots of whole blood incubated overnight with an antigenfree negative control, a phytohaemagglutinin-positive control and antigens to ESAT-6 and CFP-10.

LTBI was defined as immigrants with normal CXR and positive OFT in the absence of signs or symptoms of active TB infection, following review by a doctor in clinic. Costs were estimated for our protocol and for the NICE protocol based on NICE estimates for the cost of a CXR £23.24, TST £13.69 and OFT £25.67. ${ }^{9}$ Data analysis was carried out in Microsoft Office Excel 2003.

\section{RESULTS}

In the catchment area of the Leeds TB screening service, in 2007 there were 2902 new immigrants from countries with TB incidence $>200 / 10^{5}$ who registered with local with general practitioners. A total of 1336 were invited for screening (TST or QFT as described above), with a $32 \%$ attendance rate. Self-reported rates of HIV infection were very low (1\%). A total of 280 OFT were performed, 139 in men with an average age of 30.8 years; of these $170(60.7 \%)$ OFT were negative and 104 (37.1\%) were positive. There were $5(1.8 \%)$ indeterminate results and $1(0.3 \%)$ laboratory processing error. Of the five indeterminate results, four individuals were negative on repeat testing and one had a second indeterminate result. This individual was seen in clinic and clinically diagnosed with LTBI following a normal CXR. The individual whose sample was incorrectly processed had a negative result on second test.

Those individuals with positive OFT were invited for review in clinic. Ninety-four (90.3\%) individuals attended; of these 71 individuals had received prior BCG vaccination; 1 individual had been treated for TB in the past and had completed a 6 month course of antibiotics in Somalia; and 10 individuals had known TB contact. None reported infection with HIV. Following CXR, all individuals were diagnosed with LTBI and there were no cases of active TB identified. Sixty-four received chemoprophylaxis and 30 have been invited to attend CXR follow-up for 12 months. Of those with a positive OFT, 24 had been tested with TST, with 13 (54\%) being positive.

Overall, 221 individuals reported receiving prior BCG. The previous BCG rate was the same (79\%) in the QFT-negative and OFT-positive groups, suggesting that prior BCG is not interacting with OFT. Forty-two individuals received TST and OFT. The correlation between TST and OFT in this group was poor, with agreement in 21 (50\%) cases. Eleven individuals with a negative TST had a positive QFT, and 10 with a positive TST had a negative OFT.

Costs for screening using the Leeds protocol (OFT first) were calculated and are shown in table 1 . The total cost to screen 280 immigrants was $£ 9781.82$ ( $£ 34.94$ per immigrant) and identified 105 cases of LTBI (£93.16).

Costs which would have been incurred had we followed the NICE protocol in this group of immigrants were estimated. Based on the 42 cases who had both TST and OFT, we assumed that of those with a positive TST, $56 \%$ would have positive OFT. The numbers having both tests were too small to enable reliable estimates of the proportion of TST having positive OFT for different subgroups stratified by $\mathrm{TB}$ incidence in country of origin. The calculations are shown in table 2 . The NICE protocol would have cost an estimated total of $£ 13346.75$ ( $£ 47.67$ per immigrant) and would have identified 83 cases of LTBI ( $£ 160.81$
Table 1 Costing for screening using the Leeds protocol Leeds protocol (OFT first), 280 immigrants

Step 1: QFT

$\begin{array}{llc} & \text { Unit cost } & \text { Total cost } \\ 280 \text { QFT } & £ 25.67 & £ 7187.60 \\ 6 \text { patients had second OFT } & £ 25.67 & £ 154.02 \\ \text { 105 CXR } & £ 23.24 & £ 2440.20 \\ \text { Step 2: CXR if OFT positive (37\%)+ 1 patient with 2 indeterminate OFT } & \\ \text { Total cost for screening } & & £ 9781.82 \\ \text { LTBI cases } & & 105 \\ \text { Cost per case LTBI identified } & £ 93.16\end{array}$

CXR, chest $x$ ray; LTBI, latent tuberculosis infection; OFT, QuantiFERON-TB Gold.

per case identified). This estimate assumes that there were no "false-negative" TST. In fact, of those having both tests, 11 of 24 with positive OFT had negative TST. Allowing for this, the number of OFT done under the NICE protocol would be reduced to 83, cutting the overall cost estimate to $£ 11549.65$ ( $£ 41.25$ per immigrant) but would only identify 45 cases of LTBI ( $£ 256.66$ per case identified).

\section{DISCUSSION}

TB services are under considerable pressure to perform immigrant screening on limited resources. This is on a background of increasing incidence of $\mathrm{TB}$, largely related to cases in immigrant populations. Our service is able to screen $\sim 1200$ individuals effectively per annum. There is a need to streamline the screening process for immigrants, firstly to allow our services to operate more efficiently and effectively, and secondly to encourage participation in the screening process amongst immigrants. In our region, the aim is to screen $60 \%$ of eligible individuals, but in 2007 the attendance rate was only $32 \%$. The potential benefits of OFT over traditional TST are well recognised, but in our view the fact that only one contact with healthcare services is required is a major benefit. We have demonstrated that it is feasible to screen high risk immigrant populations using first-line OFT, although as yet we have no longitudinal data to see if this will increase attendance rates for screening.

Resources were not available to perform QFT and TST in all individuals. The cost of the NICE protocol is therefore based on an estimate of the TST-positive and OFT-positive rates in the subgroup who had both tests. This subgroup includes individuals from countries with TB incidence $200-339 / 10^{5}$ who were initially tested with TST, and those in families with very young children in whom it was not considered practical to test different

Table 2 Costing for screening using the NICE protocol NICE protocol (CXR first), 280 immigrants

Step1: CXR if age $>11$ and not pregnant

$\begin{array}{llr} & \text { Unit cost } & \text { Total cost } \\ 275 \mathrm{CXR} & £ 23.24 & £ 6393.75\end{array}$

Step 2: TST if pregnant or age $<16$ (12 individuals) or age $16-35$ from sub-Saharan Africa or from country with TB incidence $>500 / 10^{5}$ (209 individuals) 221 TST $£ 13.69$

$£ 3025.49$

Step 3: OFT if positive TST (estimated TST-positive rate 69\%) 153 QFT $£ 25.67$

$£ 3927.51$

Total cost for screening $£ 13346.75$

LTBI cases (estimated OFT-positive rate $54 \%$ ) 83

Cost per case LTBI identified

$£ 160.81$

CXR, chest $x$ ray; LTBI, latent tuberculosis infection; NICE, National Institute for Health and Clinical Excellence; QFT, QuantiFERON-TB Gold; TST, tuberculin skin test. 
family members with different tests. OFT was performed in individuals who requested OFT following the TST. This subgroup may not be representative of the entire cohort and there is, therefore, a potential for bias in the estimation of the TST-positive rate. The estimated TST-positive rate of $69 \%$ compares with a historical positive TST rate of $32 \%$ in subSaharan Africans attending our clinic for screening in $2006 .{ }^{10}$ The difference may be explained by the high proportion of those in our cohort who had both tests, with a negative TST and a positive OFT. If this proportion were consistent across the whole cohort, the estimated TST positivity rate would be $36 \%$, similar to the historical result. Applying this lower rate of TST positivity to the calculation of cost, the NICE protocol would still cost more than using the OFT test as first line and identify significantly fewer cases of LTBI.

Previous studies have demonstrated a good concordance between TST and OFT when TST is negative; however, these studies are in patients with suspected active TB or in those who are undergoing contact screening. One possible explanation is that the cut-off used in the NICE protocol for a positive TST is $15 \mathrm{~mm}$ in those who have had a prior BCG. In our group, $>75 \%$ of immigrants had received BCG. Our data suggest that cases of LTBI are potentially being missed due to the high cut-off for a positive TST. The $15 \mathrm{~mm}$ cut-off is arbitrary, and other groups have suggested a lower cut-off of $10 \mathrm{~mm}$ may be more appropriate. ${ }^{8}$ We would be interested in the experiences of other groups to see if this observation is repeated in other immigrant populations.

The low incidence of indeterminate OFT results in our study population of $1.8 \%$ compares favourably with that seen in other groups. ${ }^{6}$ The reduced rate of intermediate results could be due to increasing laboratory familiarity with the use of OFT or to differences in the study populations between different groups.

There is some limitation in our conclusions due to the change in inclusion criteria that occurred during the introduction of the new protocol. Initially we did not have the resources to offer first-line QFT to the immigrants from countries with TB incidence $200-339 / 10^{5}$. The OFT-positive rate was $34 \%$ in immigrants from countries with TB incidence $200-339 / 10^{5}$, and $43 \%$ in those from countries with TB incidence $>340 / 10^{5}$. We believe this difference would not affect our overall results and conclusions. It is interesting that our protocol identified more cases of LTBI. The increase in cases of LTBI is due to those patients who had a positive OFT but a negative TST and would therefore have been missed by the NICE protocol, and by immigrants from countries with a TB incidence of $>200 / 10^{5}$ outside sub-Saharan.
The case for selecting immigrants for screening by geography rather than by TB incidence in the country of origin seems weak, and our approach has enabled us to extend screening for LTBI to high risk countries outside Africa while still reducing the overall cost and increasing the detection of LTBI compared with the NICE protocol.

We have demonstrated that a QFT-first protocol can be carried out more cheaply than a CXR-first protocol, with a cost saving of $\sim 35 \%$. This saving is due to the reduced number of CXRs required. There would be an increase in clinic costs due to monitoring patients having chemoprophylaxis and CXR followup; however, these costs would be expected to be recouped due to the prevention of cases of active TB in those patients who receive chemoprophylaxis.

We are in the process of collecting longitudinal data to confirm that there is not an unexpectedly high incidence of active TB in those patients who had a negative OFT. Overall, we believe that using OFT blood testing followed by CXR is more effective and more cost-effective than NICE guideline for screening new entrants from high risk countries, and will continue to use this approach for immigrant screening.

\section{Competing interests None.}

Provenance and peer review Not commissioned; externally peer reviewed.

\section{REFERENCES}

1. Coker $\mathbf{R}$, Bell A, Pitman $R$, et al. Tuberculosis screening in migrants in selected European countries shows wide disparities. Eur Respir J 2006;27:801-7.

2. Mazurek G, Villarino ME. Guidelines for using the QuantiFERON-TB test for diagnosing latent mycobacterium tuberculosis infection. MMWR 2003;52:15-8.

3. Kang YA, Lee HW, Yoon HI, et al. Discrepancy between the tuberculin skin test and the whole-blood interferon gamma assay for the diagnosis of latent tuberculosis infection in an intermediate tuberculosis-burden country. JAMA 2005;293:2756-61.

4. Franken WPJ, Timmermans JF, Prins C, et al. Comparison of Mantoux and QuantiFERON TB Gold tests for diagnosis of latent tuberculosis infection in army personnel. Clin Vaccine Immunol 2007;14:477-80.

5. Diel R, Nienhaus A, Lange $C$, et al. Cost-optimisation of screening for latent tuberculosis in close contacts. Eur Resp J 2006;28:35-44.

6. Ferrara G, Losi M, D'Amico R, et al. Use in routine clinical practice of two commercial blood tests for diagnosis of infection with Mycobacterium tuberculosis: a prospective study. Lancet 2006;367:1328-34.

7. Diel R, Wrighton-Smith P, Zellweger JP. Cost-effectiveness of interferon-gamma release assay testing for the treatment of latent tuberculosis. Eur Respir $J$ 2007;30:321-32

8. Wrighton-Smith P, Zellweger JP. Direct costs of three models for the screening of latent tuberculosis infection. Eur Respir J 2006:28:45-50.

9. National Institute for Health and Clinical Excellence. Tuberculosis. Clinical diagnosis and management of tuberculosis, and measures for its prevention and control. London: NICE, 2006.

10. Datta B, Watson JP. Outcomes of new immigrant screening for tuberculosis: implications for implementation of NICE guidelines. Thorax 2006;61 (Suppl 2):ii3-ii56. 